\title{
Finite field formalism for bulk electrolyte solutions
}

\author{
Stephen J. Cox ${ }^{1}$ and Michiel Sprik ${ }^{1}$ \\ Department of Chemistry, University of Cambridge, Lensfield Road, Cambridge CB2 1EW, \\ United Kingdom ${ }^{\text {a) }}$
}

(Dated: 21 May 2020)

The manner in which electrolyte solutions respond to electric fields is crucial to understanding the behavior of these systems both at, and away from, equilibrium. The present formulation of linear response theory for such systems is inconsistent with common molecular dynamics (MD) implementations. Using the finite field formalism, suitably adapted for finite temperature MD, we investigate the response of bulk aqueous $\mathrm{NaCl}$ solutions to both finite Maxwell (E) and electric displacement (D) fields. The constant E Hamiltonian allows us to derive the linear response relation for the ionic conductivity in a simple manner that is consistent with the forces used in conventional MD simulations. Simulations of a simple point charge model of an electrolyte solution at constant $\mathbf{E}$ yield conductivities at infinite dilution within $15 \%$ of experimental values. The finite field approach also allows us to measure the solvent's dielectric constant from its polarization response, which is seen to decrease with increasing ionic strength. Comparison of the dielectric constant measured from polarization response versus polarization fluctuations enables direct evaluation of the dynamic contribution to this dielectric decrement, which we find to be small but not insignificant. Using the constant $\mathbf{D}$ formulation, we also rederive the Stillinger-Lovett conditions, which place strict constraints on the coupling between solvent and ionic polarization fluctuations.

\section{INTRODUCTION}

The role of ionic solutes in water is crucial across a broad range of scientific and technological applications. One example is the well-known Hofmeister series whereby simply changing the identity of the ions has a profound effect on protein structure and stability (see e.g. Ref. 1 for an overview). Along similar lines, tuning the electrolyte is one route to controlling self-assembly ${ }^{2-5}$ and the nucleation of molecular crystals. ${ }^{6}$ Recent work has also shown that ionic solutes can significantly impact on the ice nucleating ability of atmospherically relevant minerals, ${ }^{7}$ which is also likely to have consequences for controlling ice nucleation in cryopreservation systems. ${ }^{8}$ Electrolyte solutions are also important for energy storage applications. ${ }^{9}$ This widespread importance of electrolytes is a driving force for understanding their fundamental physical behavior. In this regard, the microscopic insight offered by molecular simulations makes them an increasingly important tool in addition to experimental studies. However, the long-ranged nature of Coulombic interactions poses major challenges for molecular simulations, especially when used in conjunction with periodic boundary conditions (PBC), which are typical for studies of condensed phase systems. Originally developed for the study of ferroelectric capacitors using Kohn-Sham density functional theory, the finite field methods developed by Stengel et $a l .{ }^{10}$ have recently been extended to finite temperature molecular dynamics simulations, ${ }^{11,12}$ and have been shown to be an effective tool for dealing with the effects of finite size when computing properties such as the capacitance of the Helmholtz layer. ${ }^{13-15}$

The purpose of this article is to take the finite field methods developed in Refs. 11-13 for the study of dielectrics and solid/electrolyte interfaces and use them to study bulk aqueous electrolyte solutions. This offers many conceptual advantages over existing theoretical treatments. In particular, we will find that the linear response (LR) relation for the static conductivity can be derived in a much simpler form than in existing formulations, ${ }^{16-18}$ and in a manner consistent with common MD implementations. We will also derive the Stillinger-Lovett (SL) conditions ${ }^{19,20}$ for bulk electrolyte solutions in the presence of an imposed electric displacement field. This approach offers a simplifying perspective that readily lends itself to an intuitive physical understanding of the observed anticorrelations between ionic and solvent polarization.

Much of our current understanding on this topic stems from the seminal works of Caillol, Levesque and Weis ${ }^{16-18}$ (CLW). The foundation of their approach is the perturbative Hamiltonian,

$$
\Delta \mathcal{H}_{\mathrm{CLW}}=-c_{\mathrm{em}}^{-1} \int \mathrm{d} \mathbf{r} \mathbf{j}_{\mathrm{ion}}(\mathbf{r}, t) \cdot \mathcal{A}(\mathbf{r}, t)+\int \mathrm{d} \mathbf{r} \rho_{\mathrm{ion}}(\mathbf{r}, t) \varphi(\mathbf{r}, t)-\int \mathrm{d} \mathbf{r} \mathbf{P}_{\mathrm{wat}}(\mathbf{r}, t) \cdot \mathcal{E}(\mathbf{r}, t),
$$

\footnotetext{
a)Electronic mail: sjc236@cam.ac.uk, ms284@cam.ac.uk
} 
where $\mathbf{j}_{\text {ion }}$ is the ionic current density, $\rho_{\text {ion }}$ is the ionic charge density, $\mathbf{P}_{\text {wat }}$ is the polarization of the solvent water molecules, and $c_{\mathrm{em}}$ is the speed of light. The vector potential $\mathcal{A}$ and the scalar potential $\varphi$ are related to the 'external' electric field by,

$$
\mathcal{E}(\mathbf{r}, t)=-c_{\mathrm{em}}^{-1} \partial_{t} \mathcal{A}(\mathbf{r}, t)-\nabla \varphi(\mathbf{r}, t) .
$$

The external field is the field that would be present in space occupied by the sample if the sample were absent, see e.g. Ref. 21. CLW work in a gauge in which $\varphi=0$. Note that this only applies to the perturbative Hamiltonian $\Delta \mathcal{H}_{\mathrm{CLW}}$, and exploits the fact that LR is gauge invariant. ${ }^{16}$ In what follows, we draw heavily on the original work of CLW, ${ }^{16-18}$ and we therefore save a detailed discussion for Sec. II, presenting instead just a brief summary of their approach here. First, $\Delta \mathcal{H}_{\mathrm{CLW}}$ is used to express the external susceptibilities $\{\boldsymbol{\chi}\}$ (see Eq. 9) in terms of the current and polarization fluctuations. Using Fulton's approach, ${ }^{22} \mathcal{E}$ is then expressed in terms of the Maxwell field $\mathbf{E}$, from which the ionic conductivity $\sigma_{\text {ion }}(\omega)$ is found from the constitutive relation, Eq. 5. The results of this procedure are fluctuation (i.e. Green-Kubo) formulas appropriate for PBC with Ewald summation, such as,

$$
\sigma_{\text {ion }}(0)=\frac{\beta}{3 \Omega} \int_{0}^{\infty} \mathrm{d} \tau\left\langle\mathbf{J}_{\text {ion }}(\tau) \cdot \mathbf{J}_{\text {ion }}(0)\right\rangle
$$

for the static ionic conductivity, where $\Omega$ is the volume of the system, $\beta=1 / k_{\mathrm{B}} T\left(k_{\mathrm{B}}\right.$ is Boltzmann's constant, and $T$ the temperature), and $\mathbf{J}_{\text {ion }}=\Omega \mathbf{j}_{\text {ion }}$ is the total ionic current. The ensemble average is understood to be taken in the absence of the perturbing field.

The CLW formulation of LR is the basis of many simulation studies of conducting liquids, and is used to derive the Einstein-Helfand relation for the static conductivity ${ }^{23}$ as well as to compute dielectric spectra. ${ }^{23-28}$ However, $\Delta \mathcal{H}_{\mathrm{CLW}}$ is not a Hamiltonian from which the forces required for MD simulations can be readily derived. This is a rather disconcerting aspect of this LR formulation, especially if we wish to drive the simulated system with a perturbing field in a rigorous manner. This is one of the issues we address in this article. This also leads to practical benefits, as it allows us to directly measure the solvent dielectric constant and ionic conductivity directly from the response to a finite field, rather than relying on Green-Kubo formulas that can be difficult to converge for electrolyte systems (see Fig. S8). Such an approach also allows us to directly measure the 'dynamic contribution' to the dielectric decrement as the difference between the dielectric constant measured from the solvent response, to that from its polarization fluctuations at equilibrium. Since its conception by Hubbard and co-workers, ${ }^{29-31}$ and later Felderhof, ${ }^{32}$ the understanding and calculation of this dynamic contribution has proved challenging for both theory and simulation. Based on a linear hydrodynamics model, Chandra et al..$^{33}$ derived that this dynamic decrement strictly vanishes for spherical ions in a solvent of arbitrary molecular symmetry. Later simulations from Chandra, ${ }^{34}$ however, suggested that the dynamic contribution was in fact nonzero, but still approximately two orders of magnitude smaller than the equilibrium contributions to the dielectric decrement. Results from our simulations corroborate this later finding that the dynamic contribution is finite, although we will see that it is significantly larger than suggested by Chandra.

In addition to providing a means to simulating systems at constant Maxwell field $\mathbf{E}$, the recent developments of Refs. 11-13 also outline a procedure for performing simulations at fixed electric displacement D. Methodologically, this is perhaps a more significant theoretical advance than the constant $\mathbf{E}$ ensemble. As such, the response of bulk electrolyte solutions to constant $\mathbf{D}$ fields are not widely studied with computer simulation. However, we note that Caillol and co-workers realized that $\epsilon^{\prime}=0$ is a relevant boundary condition in a formulation in which the sample is surrounded by a medium of dielectric constant $\epsilon^{\prime} \cdot{ }^{17,18,35}$ This was later identified as corresponding to a $\mathbf{D}=\mathbf{0}$ ensemble, ${ }^{11-13}$ which is elaborated upon in Sec. II B. One example of previous work that has explicitly used the constant $\mathbf{D}$ formulation for electrolyte solutions is that of Pache and Schmid ${ }^{36}$ who investigated the concentration dependence of the solvent dielectric constant of various electrolyte solutions, and reported severe dielectric saturation at moderate field strengths (e.g. the dielectric constant decreases by approx. $50 \%$ for $\mathbf{D}=2 \mathrm{~V} / \AA$ ). These results are discussed in the context of the SL conditions below.

The rest of the article is as follows. In Sec. II we give a general outline of the relevant theory, with derivations of the LR relation for the static conductivity given in Sec. II A, and the SL conditions in Sec. II B. Simulation methods are given in Sec. III. In Sec. IV we present results from our simulations, with the response to finite $\mathbf{E}$ and finite $\mathbf{D}$ given in Secs. IV A and IV B, respectively. We end with a summary in Sec. V.

\section{THEORETICAL OUTLINE}

Central to this work are the constitutive relations that relate the Maxwell field $\mathbf{E}$ to the water polarization $\mathbf{P}_{\text {wat }}$ and the ionic current density $\mathbf{j}_{\text {ion }}$. They are,

$$
\begin{aligned}
\mathbf{P}_{\text {wat }}(\mathbf{r}, \omega) & =\frac{\epsilon_{\mathrm{wat}}(\omega)-1}{4 \pi} \mathbf{E}(\mathbf{r}, \omega), \\
\mathbf{j}_{\text {ion }}(\mathbf{r}, \omega) & =\sigma_{\text {ion }}(\omega) \mathbf{E}(\mathbf{r}, \omega),
\end{aligned}
$$

where $\epsilon_{\text {wat }}$ is the dielectric constant of the solvent water, and $\sigma_{\text {ion }}$ is the ionic conductivity. $\mathbf{P}_{\text {wat }}$ and $\mathbf{j}_{\text {ion }}$ are understood to be ensemble averages. For notational convenience, we omit angled brackets when this is clear from 
context, although the standard notation ' $\langle\cdot\rangle$ ' will be used to denote such ensemble averages when required. In writing Eqs. 4 and 5, the possibility of time dependent fields has been considered, with $\omega$ the frequency of oscillation. Throughout the article, the Fourier transform in the time domain is defined as e.g.,

$$
\mathbf{P}_{\text {wat }}(\mathbf{r}, \omega)=\int_{-\infty}^{\infty} \mathrm{d} t \mathbf{P}_{\text {wat }}(\mathbf{r}, t) \exp (i \omega t)
$$

(Note that this is a full transform over the time domain. For the susceptibilities this would be a half-transform.) From a molecular simulation, one has direct access to $\mathbf{P}_{\text {wat }}$ and $\mathbf{j}_{\text {ion }}$. Experimentally, however, it is the total current $\mathbf{j}=\mathbf{j}_{\text {ion }}+\partial_{t} \mathbf{P}_{\text {wat }}$ that is measured. Its relation to $\mathbf{E}$ is,

$$
\mathbf{j}(\omega)=\sigma_{\mathrm{T}}(\omega) \mathbf{E}(\omega),
$$

with,

$$
\sigma_{\mathrm{T}}(\omega)=\sigma_{\mathrm{ion}}(\omega)-\frac{i \omega}{4 \pi}\left[\epsilon_{\mathrm{wat}}(\omega)-1\right] .
$$

Note that in writing Eqs. 4, 5 and 7, we have implicitly assumed that the response is local.

In addition to these constitutive relations, it is also desirable to relate $\mathbf{P}_{\text {wat }}, \mathbf{j}_{\text {ion }}$ and $\mathbf{j}$ to the perturbing field. For example,

$$
4 \pi \mathbf{P}_{\text {wat }}(\omega)=\chi_{P_{\mathrm{w}} P}(\omega) * \mathbf{E}_{0}(\omega),
$$

Similar relations are also defined for $\mathbf{j}_{\text {ion }}, \mathbf{j}$ and the total polarization $\mathbf{P}=\mathbf{P}_{\text {ion }}+\mathbf{P}_{\text {wat }}$, each with its own susceptibility $\left(\chi_{j_{\mathrm{i}} P}, \boldsymbol{\chi}_{j P}\right.$ and $\chi_{P P}$, respectively), which will be referred to collectively as $\{\boldsymbol{\chi}\} .{ }^{37}$ Eq. 9 also introduces the shorthand notation in which ' $*$ ' denotes both tensor contraction and spatial convolution, i.e.,

$$
\chi(\omega) * \mathbf{E}_{0}(\omega)=\sum_{\alpha \gamma} \int \mathrm{d} \mathbf{r}^{\prime} \chi_{\alpha \gamma}\left(\left|\mathbf{r}-\mathbf{r}^{\prime}\right|, \omega\right) E_{0, \gamma}\left(\mathbf{r}^{\prime}, \omega\right) \hat{\mathbf{e}}_{\alpha},
$$

where $\alpha$ and $\gamma$ denote components of a Cartesian coordinate system, and $\hat{\mathbf{e}}_{\alpha}$ is the unit vector along direction $\alpha$. Our choice of notation ' $\mathbf{E}_{0}$ ' for the perturbing field requires some clarification. For consistency with the finite field Hamiltonians (see Eqs. 13 and 19), we ultimately wish to identify $\mathbf{E}_{0}$ with either the Maxwell field $\mathbf{E}$ or displacement field $\mathbf{D}$. For the constant $\mathbf{E}$ ensemble, this is straightforward. Imposing constant $\mathbf{D}$, on the other hand, gives rise to subtleties which motivates the use of the following general Hamiltonian to formulate the LR relations,

$$
\begin{aligned}
\mathcal{H}\left(\mathbf{r}^{N}, \mathbf{p}^{N}\right) & =\mathcal{H}_{0}\left(\mathbf{p}^{N}, \mathbf{r}^{N}\right)-\int \mathrm{d} \mathbf{r} \mathbf{E}_{0} \cdot \mathbf{P}\left(\mathbf{r}^{N}\right), \\
& =\mathcal{H}_{0}\left(\mathbf{r}^{N}, \mathbf{p}^{N}\right)-\Omega \mathbf{E}_{0} \cdot \mathbf{P}\left(\mathbf{r}^{N}\right) .
\end{aligned}
$$

In going from the first to the second lines, we note that we only consider uniform fields. $\mathcal{H}_{0}$ is the Hamiltonian when $\mathbf{E}_{0}=\mathbf{0}$. Both $\mathbf{E}_{0}$ and $\mathcal{H}_{0}$ depend upon the choice of boundary conditions.

\section{A. Linear response relation for the static conductivity}

As discussed in Sec. I, the CLW approach to LR is based on the perturbative Hamiltonian given by Eq. 1 . This couples $\mathbf{j}_{\text {ion }}$ to the vector potential $\mathcal{A}$. In comparison, within the finite field formulation, the Hamiltonian for an imposed, uniform, although potentially timedependent, $\mathbf{E}$ reads,

$$
\mathcal{H}_{\mathbf{E}}\left(\mathbf{r}^{N}, \mathbf{p}^{N}\right)=\mathcal{H}_{\mathrm{PBC}}\left(\mathbf{r}^{N}, \mathbf{p}^{N}\right)-\frac{\Omega}{8 \pi}|\mathbf{E}|^{2}-\Omega \mathbf{E} \cdot \mathbf{P}\left(\mathbf{r}^{N}\right),
$$

where $\mathcal{H}_{\mathrm{PBC}}$ is a 'standard' Hamiltonian used in MD simulation, which comprises all interatomic interactions, with electrostatic interactions calculated with Ewald summation (or one of its mesh based variants). Again we emphasize that $\mathbf{E}$ is the Maxwell field. The total polarization is defined as the time integral of the current,

$$
\mathbf{P}\left(\mathbf{r}^{N}\right)=\frac{1}{\Omega} \sum_{i} q_{i} \mathbf{r}_{i}(t)
$$

where $q_{i}$ is the charge of atom $i$, whose position at time $t$ is denoted by $\mathbf{r}_{i}(t)$. Crucially, the sum includes both the atoms of the solvent molecules and the charged ions. The polarization that couples to $\mathbf{E}$ is the itinerant polarization. When an ion leaves the primary simulation cell, we follow its position out of the box, and it is these coordinates that enter into the sum in Eq. 14. Use of the itinerant polarization in molecular simulations with PBC is not new; as noted by Caillol, it is the itinerant polarization that enters naturally in the Ewald sum, and satisfies key statistical mechanical properties such as the SL sum rules. ${ }^{35}$ Although Eq. 13 was first derived on thermodynamic grounds, we stress that it is a full microscopic Hamiltonian. This was recently formalized in Ref. 38 where is was derived from an extended Lagrangian based on arguments of theoretical mechanics. In fact, the finite field Hamiltonians given by Eqs. 13 and 19 can be obtained by a Power-Zienau gauge transformation ${ }^{39}$ from the minimal coupling Hamiltonian used by CLW, with the restriction that $\mathbf{E}$ and $\mathbf{D}$ must be uniform. These Hamiltonians also respect the inherently multivalued nature of the polarization under PBC. ${ }^{13,38}$

With the form of $\mathcal{H}_{\mathbf{E}}$ given by Eq. 13, we can readily identify the perturbing field $\mathbf{E}_{0}$ with the Maxwell field E (see Eq. 12). Note that the second term on the right hand side of Eq. 13 is constant for a given $\mathbf{E}$; it is required to ensure that the electric enthalpy at constant $\mathbf{E}$ and electric internal energy at constant $\mathbf{D}$ are each other's Legendre transforms. ${ }^{10,11,38,40}$ The derivation of the LR relation for $\sigma_{\text {ion }}(0)$ now follows standard textbook arguments. ${ }^{41}$ Taking $\mathbf{E}$ to be a monochromatic field of frequency $\omega$, aligned along the $x$ direction for convenience, the total current is,

$$
\left\langle J_{x}(t)\right\rangle=\chi_{J M}(\omega) E \exp (-i \omega t)
$$


with,

$$
\chi_{J M}(\omega)=\beta \int_{0}^{\infty} \mathrm{d} \tau\left\langle J_{x}(\tau) J_{x}(0)\right\rangle \exp (i \omega \tau) .
$$

Comparing to Eq. 7 we find,

$$
\sigma_{\mathrm{T}}(\omega)=\frac{\beta}{\Omega} \int_{0}^{\infty} \mathrm{d} \tau\left\langle J_{x}(\tau) J_{x}(0)\right\rangle \exp (i \omega \tau)
$$

Exploiting the isotropy of the system, and taking the static limit (see Eq. 8) gives,

$$
\lim _{\omega \rightarrow 0} \sigma_{\mathrm{T}}(\omega)=\sigma_{\text {ion }}(0)=\frac{\beta}{3 \Omega} \int_{0}^{\infty} \mathrm{d} \tau\langle\mathbf{J}(\tau) \cdot \mathbf{J}(0)\rangle .
$$

Aside from being a decidedly simpler derivation than that based on $\Delta \mathcal{H}_{\mathrm{CLW}},{ }^{16-18}$ the pleasing aspect of the above derivation is that $\mathcal{H}_{\mathbf{E}}$ is the same Hamiltonian used to derive the forces for $\mathrm{MD}$ simulations. It amounts to simply adding a force $\mathbf{f}_{\mathbf{E}}=q_{i} \mathbf{E}$ to each atom $i$ in the simulation. We exploit this fact in our simulations, which are presented in Sec. IV A. Note that the use of uniform fields is crucial to this formulation.

\section{B. Stillinger-Lovett conditions}

The Stillinger-Lovett conditions are a statement that the mobile ions completely screen the solvent from slowly-varying, static fields. ${ }^{17-20,35,42-44}$ From the finite field Hamiltonian for constant displacement field D,

$$
\mathcal{H}_{\mathbf{D}}\left(\mathbf{r}^{N}, \mathbf{p}^{N}\right)=\mathcal{H}_{\mathrm{PBC}}\left(\mathbf{r}^{N}, \mathbf{p}^{N}\right)+\frac{\Omega}{8 \pi}\left|\mathbf{D}-4 \pi \mathbf{P}\left(\mathbf{r}^{N}\right)\right|^{2},
$$

it is possible to formulate the SL conditions in a manner that ultimately avoids invoking abstract cavity relations. In what follows, we will work in an ensemble in which the displacement field is fixed in all three Cartesian directions, $\mathbf{D}=D_{x} \hat{\mathbf{x}}+D_{y} \hat{\mathbf{y}}+D_{z} \hat{\mathbf{z}}$. We begin by stating the $\mathrm{LR}$ relation for the total polarization in response to D,

$$
\langle\mathbf{P}\rangle_{\mathbf{D}}=\frac{\beta \Omega}{3}\left\langle|\delta \mathbf{P}|^{2}\right\rangle_{0} \mathbf{D}
$$

where $\delta \mathbf{P}=\mathbf{P}-\langle\mathbf{P}\rangle_{0}$. The subscript ' 0 ' indicates averages taken at $\mathbf{D}=\mathbf{0}$. From the definition of the polarizability, the fluctuations in the total dipole moment $\mathbf{M}=\Omega \mathbf{P}$ are related to the dielectric constant, ${ }^{12}$

$$
\frac{4 \pi \beta}{3 \Omega}\left\langle|\delta \mathbf{M}|^{2}\right\rangle_{0}=\left(\frac{\epsilon-1}{\epsilon}\right)
$$

For conducting electrolyte systems, we are interested in the limit $\epsilon \rightarrow \infty$,

$$
\frac{4 \pi \beta}{3 \Omega}\left\langle|\delta \mathbf{M}|^{2}\right\rangle_{0}=1
$$

Substituting Eq. 22 into Eq. 20 gives $4 \pi\langle\mathbf{P}\rangle_{\mathbf{D}}=\mathbf{D}$. From the fundamental equation of Maxwell's theory of dielectrics, $\mathbf{D}=\mathbf{E}+4 \pi \mathbf{P}$, we find,

$$
\langle\mathbf{E}\rangle=0
$$

This simply reflects the fact that at equilibrium, the total electric field inside a conducting medium vanishes. From Eqs. 4 and 5 we find that, $\left\langle\mathbf{P}_{\text {wat }}\right\rangle_{\mathbf{D}}=\left\langle\mathbf{j}_{\text {ion }}\right\rangle_{\mathbf{D}}=\mathbf{0}$.

Following the discussion at the end of Sec. II, we now consider the general Hamiltonian given by Eq. 12. In this case, the external susceptibilities $\{\boldsymbol{\chi}\}$ are related to the time correlation functions of the system at $\mathbf{E}_{0}=\mathbf{0}$,

$$
\begin{aligned}
\chi_{P_{\mathrm{w}} P}(\omega) & =4 \pi \beta\left\langle\mathbf{P}_{\text {wat }}(t) \mathbf{j}(0)\right\rangle_{\omega}, \\
\chi_{j_{\mathrm{i}} P}(\omega) & =4 \pi \beta\left\langle\mathbf{j}_{\text {ion }}(t) \mathbf{j}(0)\right\rangle_{\omega}, \\
\chi_{j P}(\omega) & =4 \pi \beta\langle\mathbf{j}(t) \mathbf{j}(0)\rangle_{\omega},
\end{aligned}
$$

where $\langle\cdot\rangle_{\omega}$ denotes the Fourier-Laplace transform. (In writing Eqs. 24-26, we have used the fact that the average polarization and current densities formally vanish at zero field.) Noting that $\mathbf{j}=\mathbf{j}_{\text {ion }}+\mathbf{j}_{\text {wat }}$, exploiting well-known properties of time correlations functions, and integrating by parts a number of times, we find,

$$
\begin{aligned}
& \chi_{P_{\mathrm{w}} P}(\omega)=4 \pi \beta\left[\left\langle\mathbf{P}_{\mathrm{wat}} \mathbf{P}_{\mathrm{wat}}\right\rangle+\left\langle\mathbf{P}_{\mathrm{wat}} \mathbf{P}_{\text {ion }}\right\rangle+i \omega\left\langle\mathbf{P}_{\mathrm{wat}}(t) \mathbf{P}_{\mathrm{ion}}(0)\right\rangle_{\omega}+i \omega\left\langle\mathbf{P}_{\mathrm{wat}}(t) \mathbf{P}_{\mathrm{wat}}(0)\right\rangle_{\omega}\right] \\
& \chi_{j_{\mathrm{i}} P}(\omega)=-4 \pi \beta i \omega\left[\left\langle\mathbf{P}_{\mathrm{ion}} \mathbf{P}_{\text {ion }}\right\rangle+\left\langle\mathbf{P}_{\mathrm{ion}} \mathbf{P}_{\mathrm{wat}}\right\rangle+i \omega\left\langle\mathbf{P}_{\mathrm{ion}}(t) \mathbf{P}_{\mathrm{wat}}(0)\right\rangle_{\omega}+i \omega\left\langle\mathbf{P}_{\mathrm{ion}}(t) \mathbf{P}_{\mathrm{ion}}(0)\right\rangle_{\omega}\right] \\
& \chi_{j P}(\omega)=\boldsymbol{\chi}_{j_{\mathrm{i}} P}(\omega)-i \omega \boldsymbol{\chi}_{P_{\mathrm{w}} P}(\omega)
\end{aligned}
$$

As the time derivative of the polarization gives the cur-

$$
\begin{aligned}
\text { rent, } \mathbf{j}(\omega) & =-i \omega \mathbf{P}(\omega) \\
\chi_{P P}(\omega) & =\frac{i}{\omega} \chi_{j P}(\omega)=\left[\chi_{P_{\mathrm{w}} P}(\omega)+\frac{i}{\omega} \chi_{j_{\mathrm{i}} P}(\omega)\right] .
\end{aligned}
$$


Importantly, for static fields,

$$
\chi_{P P}(\omega=0)=\left[\chi_{P_{\mathrm{w}} P}(\omega=0)+\lim _{\omega \rightarrow 0} \frac{i}{\omega} \chi_{j_{\mathrm{i}} P}(\omega)\right] .
$$

As both $\left\langle\mathbf{P}_{\text {wat }}\right\rangle_{\mathbf{D}}$ and $\left\langle\mathbf{j}_{\text {ion }}\right\rangle_{\mathbf{D}}$ vanish, consistency demands that,

$$
\begin{array}{r}
\int_{\Omega} \mathrm{d} \mathbf{r}^{\prime} \chi_{P_{\mathrm{w}} P}\left(\mathbf{r}, \mathbf{r}^{\prime}, \omega=0\right)=\mathbf{0} \\
\int_{\Omega} \mathrm{d} \mathbf{r}^{\prime} \chi_{j_{\mathrm{i}} P}\left(\mathbf{r}, \mathbf{r}^{\prime}, \omega=0\right)=\mathbf{0}
\end{array}
$$

From Eqs. 27 and 32 we derive the first SL sum rule,

$$
\frac{4 \pi \beta}{3 \Omega}\left[\left\langle\left.\mathbf{M}_{\mathrm{wat}}\right|^{2}\right\rangle+\left\langle\mathbf{M}_{\mathrm{wat}} \cdot \mathbf{M}_{\mathrm{ion}}\right\rangle\right]=0 .
$$

According to Eq. 28, $\chi_{j_{\mathrm{i}} P}(\omega=0)=0$, and Eq. 33 therefore contains no useful information. In order to proceed, we will follow Fulton's approach ${ }^{22}$ to relate $\mathbf{E}$ to $\mathbf{E}_{0}$ and $\mathbf{P}$,

$$
\mathbf{E}=\mathbf{E}_{0}+4 \pi \mathbf{G}_{0} * \mathbf{P}
$$

where $\mathbf{G}_{0}$ is the Green's function for the constant $\mathbf{D}$ ensemble. That is, $\mathbf{G}_{0}\left(\mathbf{r}, \mathbf{r}^{\prime}\right) * \boldsymbol{\mu}^{\prime}$ is the electric field at $\mathbf{r}$ caused by a dipole $\boldsymbol{\mu}^{\prime}$ at position $\mathbf{r}^{\prime}$ under PBC at constant D. As noted in Sec. I, it has already been established that the $\mathbf{D}=\mathbf{0}$ Hamiltonian has the same structure as that derived from a reaction field approach in which the surrounding medium has vanishing dielectric constant $\left(\epsilon^{\prime}=0\right)$. We can exploit this fact to obtain $\mathbf{G}_{0}$, which is readily achieved by following Ref. 45. For the isotropic systems considered here, we only require its trace at $\omega=0$,

$$
\operatorname{Tr} \mathbf{G}_{0}\left(\mathbf{r}, \mathbf{r}^{\prime}, \omega=0\right)=-\left[\delta_{\mathrm{EW}}\left(\mathbf{r}-\mathbf{r}^{\prime}\right)+\frac{2}{\Omega}\right],
$$

where $\delta_{\mathrm{EW}}\left(\mathbf{r}-\mathbf{r}^{\prime}\right)$ is the periodic Dirac delta-function. We now substitute $4 \pi \mathbf{P}=\chi_{P P} * \mathbf{E}_{0}$ into Eq. 35, which after setting $\mathbf{E}=\mathbf{0}$ gives,

$$
\int_{\Omega} \mathrm{d} \mathbf{r}^{\prime} \int_{\Omega} \mathrm{d} \mathbf{r}^{\prime \prime} \mathbf{G}_{0}\left(\mathbf{r}, \mathbf{r}^{\prime}\right) \cdot \chi_{P P}\left(\mathbf{r}^{\prime}, \mathbf{r}^{\prime \prime}, \omega=0\right)=\mathbf{- 1} .
$$

Equations 31 and 32 allow us to write the left hand side as,

$$
\int_{\Omega} \mathrm{d} \mathbf{r}^{\prime} \int_{\Omega} \mathrm{d} \mathbf{r}^{\prime \prime} \mathbf{G}_{0}\left(\mathbf{r}, \mathbf{r}^{\prime}\right) \cdot \lim _{\omega \rightarrow 0} \frac{i}{\omega} \chi_{j_{\mathrm{i}} P}\left(\mathbf{r}^{\prime}, \mathbf{r}^{\prime \prime}, \omega\right) .
$$

The fact that the system is isotropic allows us to write $\chi_{j_{\mathrm{i}} P}=\chi_{j_{\mathrm{i}} P} \mathbf{1}$, where $\chi_{j_{\mathrm{i}} P}$ is a scalar. Moreover, as we are only concerned with uniform fields, only the zero mode of the susceptibility contributes upon integration. Thus, upon taking the trace we obtain,

$$
-\lim _{\omega \rightarrow 0} \int_{\Omega} \mathrm{d} \mathbf{r}^{\prime} \int_{\Omega} \mathrm{d} \mathbf{r}^{\prime \prime}\left[\delta_{\mathrm{EW}}\left(\mathbf{r}-\mathbf{r}^{\prime}\right)+\frac{2}{\Omega}\right] \frac{i}{\omega} \chi_{j_{\mathrm{i}} P}\left(\mathbf{r}^{\prime}, \mathbf{r}^{\prime \prime}, \omega\right)=-\lim _{\omega \rightarrow 0} \frac{3}{\Omega} \int_{\Omega} \mathrm{d} \mathbf{r}^{\prime} \int_{\Omega} \mathrm{d} \mathbf{r}^{\prime \prime} \frac{i}{\omega} \chi_{j_{\mathrm{i}} P}\left(\mathbf{r}^{\prime}, \mathbf{r}^{\prime \prime}, \omega\right)
$$

Using our expression for $\chi_{j_{\mathrm{i}} P}$ in terms of the time correlation functions (Eq. 28), and after taking the trace of the unit tensor (see Eq. 37), we find,

$$
\frac{4 \pi \beta}{\Omega} \int_{\Omega} \mathrm{d} \mathbf{r}^{\prime} \int_{\Omega} \mathrm{d} \mathbf{r}^{\prime \prime}\left[\left\langle\mathbf{P}_{\text {ion }} \cdot \mathbf{P}_{\text {ion }}\right\rangle+\left\langle\mathbf{P}_{\text {ion }} \cdot \mathbf{P}_{\text {wat }}\right\rangle\right]=3
$$

or,

$$
\frac{4 \pi \beta}{3 \Omega}\left[\left\langle\left|\mathbf{M}_{\text {ion }}\right|^{2}\right\rangle+\left\langle\mathbf{M}_{\text {ion }} \cdot \mathbf{M}_{\text {wat }}\right\rangle\right]=1
$$

This is the second SL condition. As a sanity check, addition of Eqs. 34 and 41 recovers the LR condition for the total polarization fluctuations, Eq. 22.

The above derivation is broadly similar to that of Caillol, Levesque and Weis. ${ }^{17}$ The main difference lies in the fact that we have associated $\mathbf{G}_{0}$ as the Greens' function for the constant-D ensemble. The significance of this statement becomes clear when we attempt to perturb the system with finite $\mathbf{E}_{0}$, which we show below that we can identify with D. In the original CLW formulation, the perturbing field is $\mathcal{E}$ (Eq. 1), which is then associated with the 'cavity field'. ${ }^{17}$ The drawback of this approach is that one cannot readily identify the molecular forces associated with the perturbing field. In contrast, the current formulation allows us to readily derive the appropriate forces from $\mathcal{H}_{\mathbf{D}}$ (Eq. 19), and thus probe the system's response to finite values of $\mathbf{D}$. Crucial to the current formulation is that we have accounted for the ions' charge in the intinerant polarization, and the only source of $\mathbf{D}$ is the charge on the electrodes at infinity. The extent to which the SL conditions remain valid at finite $\mathbf{D}$ places this interpretation on a firm statistical mechanical basis that can be verified empirically by simulation, beyond the theoretical arguments in Ref. 38.

In passing, we note that in a later paper, ${ }^{35}$ Caillol suggests that the itinerant polarization behaves like an independent harmonic oscillator (Eq. 22), and in that sense, the total SL condition is a mere consequence of energy equipartition. Such an interpretation is misleading when 
considered more generally. The constant D Hamiltonian is oblivious to properties of the system; it simply does not know if it governs the dynamics of a conductor or a dielectric. Arguments based on energy equipartition would suggest Eq. 22 holds in all cases. This, however, would violate the LR relations for dielectrics (see Eq. 21), which have previously been shown to work well for describing bulk water. ${ }^{11}$

What remains is to establish that we can indeed associate the perturbing field $\mathbf{E}_{0}$ with the displacement field D. To this end, we expand the quadratic term in $\mathcal{H}_{\mathbf{D}}$ (Eq. 19),

$$
\mathcal{H}_{\mathbf{D}}=\mathcal{H}_{\mathrm{PBC}}+2 \pi \Omega|\mathbf{P}|^{2}+\frac{\Omega}{8 \pi}|\mathbf{D}|^{2}-\Omega \mathbf{D} \cdot \mathbf{P} .
$$

Comparing to the general LR Hamiltonian (Eq. 12), we see that unlike the constant-E ensemble, $\mathcal{H}_{0}$ (the Hamiltonian at $\mathbf{D}=\mathbf{0}$ ) contains a term quadratic in the total polarization. This is, of course, the origin of the distinctly different fluctuations in the two ensembles. ${ }^{11}$ Once we acknowledge the belonging of the $|\mathbf{P}|^{2}$ term to $\mathcal{H}_{0}$, direct comparison of Eqs. 12 and 19 allows us to identify $\mathbf{D}$ with $\mathbf{E}_{0}$.

We end this section with a comment on the coupling between ionic and solvent polarization fluctuations. The SL conditions place strict requirements on the behavior of the electrolyte solution. In particular, it is clear that $\left\langle\delta \mathbf{M}_{\text {wat }} \cdot \delta \mathbf{M}_{\text {ion }}\right\rangle<0$ if Eq. 34 is to be satisfied. In other words, the ionic and water polarization fluctuations are anticorrelated. The finite field method framework provides a useful physical interpretation for this result. To this end, we consider 'hybrid' boundary conditions in which the displacement field is only set along one of the Cartesian directions, e.g. $\mathbf{D}=D_{x} \hat{\mathbf{x}}$, while tin-foil boundary conditions are used in the transverse directions $\left(E_{y}=E_{z}=0\right)$. In this constant- $D_{x}$ ensemble, the behavior is analogous to that of a system between a pair of electrodes whose equal-and-opposite charges are fixed. ${ }^{11}$ At equilibrium, the ions relax such that $4 \pi\left\langle P_{x \text {,ion }}\right\rangle=D_{x}$, and $\left\langle P_{x \text {,wat }}\right\rangle=0$. This is depicted schematically in Fig. 1(a). Owing to thermal motion, the ions will fluctuate around their equilibrium configurations such that at any instant $4 \pi P_{x}$,ion $\neq D_{x}$, and the field is not fully screened, as shown in Fig. 1 (b). It is reasonable to assume that the timescale on which the solvent reorganizes is faster than that for the ions to relax back toward equilibrium. Consequently, it is expected that a transient polarization of the water will be observed, and aligned in the opposite direction to the transient fluctuation in the ionic polarization. While this parallel plate capacitor analogy cannot be rigorously extended to the ensemble in which $\mathbf{D}=D_{x} \hat{\mathbf{x}}+D_{y} \hat{\mathbf{y}}+D_{z} \hat{\mathbf{z}},{ }^{11}$ we show in the S.I. that empirically the SL conditions are still satisfied in the case of hybrid boundary conditions. Thus, the underlying principle - that fluctuations in the ionic polarization lead to a transient incomplete screening with associated solvent response - appears to hold true in both ensembles.

\section{METHODS}

The system we consider is aqueous sodium chloride $(\mathrm{NaCl})$ with concentrations in the range $0.05 \lesssim c \lesssim$ 8.65 M, modeled under 3D PBC with Ewald summation, and in the absence of extended interfaces. For $c>0.4 \mathrm{M}$ each simulation comprised $256 \mathrm{SPC} / \mathrm{E}^{46}$ water molecules, corresponding to a number of ion pairs between two and forty for the concentration ranges investigated. The cubic dimension of the simulation cell was $L=19.73 \AA$, and the volume $\Omega=L^{3}$ was the same for all $c>0.4 \mathrm{M}$. This corresponds to a constant number density of water of $33.33 \times 10^{-3} \AA^{-3}$. Consequently, the pressure increases dramatically as the concentration of the solution increases. However, we show in the S.I. that similar behavior is observed when we adjust the size of the simulation cell such that the average pressure is approximately constant. For the two lowest concentrations investigated, $c \approx 0.05 \mathrm{M}$ and $c \approx 0.11 \mathrm{M}$, simulations were performed with 1024 and 2048 water molecules, respectively, with two ion pairs in both cases. The cell lengths were $L=31.32 \AA(0.05 \mathrm{M})$ and $L=39.46 \AA(0.11 \mathrm{M})$. As one of the principal aims of this study is to demonstrate the application of the finite field methods to bulk electrolyte solutions, we are content with limiting ourselves to relatively small simulation cells for the sake of computational efficiency. While we believe our simulations to be sufficient for our current purposes, we have not investigated the potential effects of finite system size. The ion-ion and ion-water interactions were described with a Lennard-Jones potential and point charges, using the parameters derived by Joung and Cheatham. ${ }^{47}$ Each simulation was approximately $50 \mathrm{~ns}$.

For all simulations we used the LAMMPS simulation package. ${ }^{48}$ The particle-particle particle-mesh Ewald method was used to account for long-ranged interactions ${ }^{49}$ with parameters chosen such that the root mean square error in the forces were a factor $10^{5}$ smaller than the force between two unit charges separated by a distance of $1.0 \AA{ }^{50}$ Dynamics were propagated using a velocity-Verlet algorithm with a time step of either $1 \mathrm{fs}$ or $2 \mathrm{fs}$. The temperature was maintained using a Nosè-Hoover thermostat ${ }^{51,52}$ at $T=298 \mathrm{~K}$. The geometry of the water molecules was maintained using the RATTLE algorithm. ${ }^{53}$ The constant-E Hamiltonian is implemented as standard in LAMMPS. On the other hand, the constant-D Hamiltonian was implemented 'in-house'. ${ }^{54}$

\section{SIMULATION RESULTS}

\section{A. Electrolyte response to constant $\mathbf{E}$}

In Sec. II A, we presented a straightforward derivation of the LR relation for $\sigma_{\text {ion }}(0)$ within the finite field formalism. In this section, we make use of the fact that $\mathcal{H}_{\mathbf{E}}$ is a fully microscopic Hamiltonian, and explicitly simulate $\mathrm{NaCl}$ solutions at finite E. As mentioned previously, 

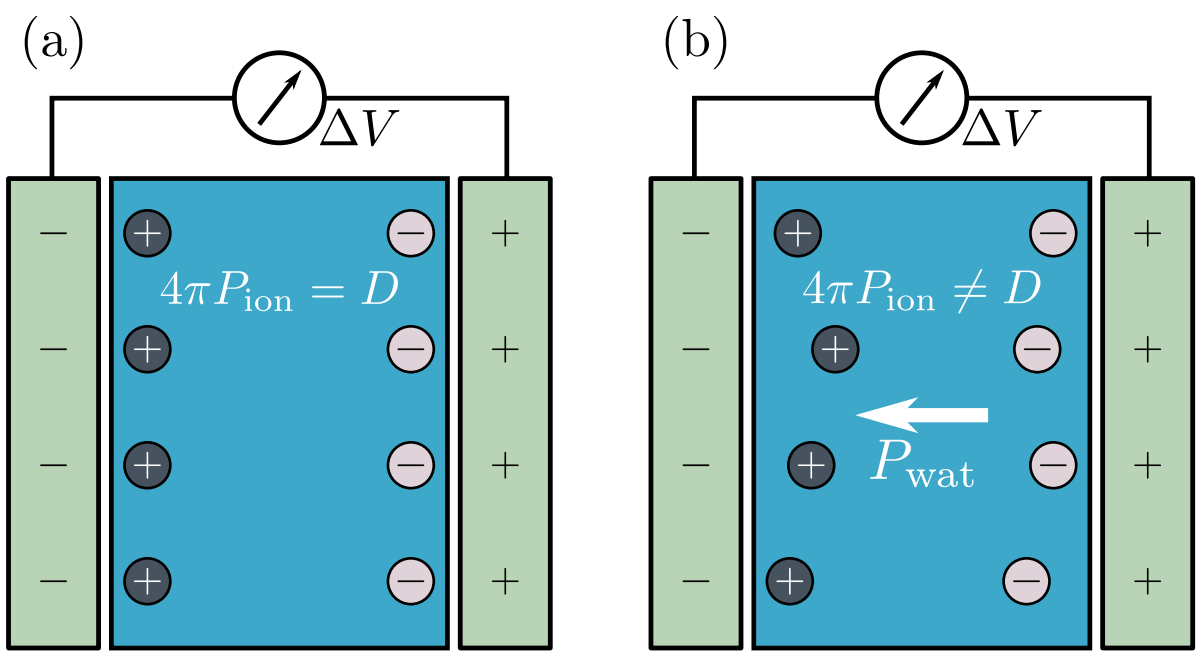

FIG. 1. Schematic of electrolyte behavior at constant $\mathbf{D}=D_{x} \hat{\mathbf{x}}$, which can be modeled as a pair of electrodes held at constant charge. (a) At equilibrium, the ions relax such that $4 \pi\left\langle\mathbf{P}_{\text {ion }}\right\rangle=\mathbf{D}$. This total screening of the $\mathbf{D}$ field by the ions means the average solvent polarization vanishes. (b) A thermal fluctuation displaces the ions from their equilibrium configuration, and the field is no longer completely screened. A transient solvent polarization is observed, which is in the opposite direction to the ionic polarization fluctuation.

if an atom $i$ has a charge $q_{i}$, this amounts to simply applying a force $\mathbf{f}_{\mathbf{E}}=q_{i} \mathbf{E}$ to that atom. Although this is what one might guess naively, applications of this approach to bulk electrolyte solutions are surprisingly scarce, although there are a number of examples in the biophysical literature for calculating ionic fluxes through membranes (see e.g. Refs. 55-57). In Fig. 2 (a) we show the time evolution of $P_{x \text {,ion }}$, the $x$ component of ionic polarization, for $1 \mathrm{M} \mathrm{NaCl}$. As the itinerant polarization is the time integral of the current density, we infer from Fig. 2 (a) that we have reached a non-equilibrium steady state for each value of $E_{x}$. Having obtained the time evolution of $P_{x, \text { ion }}$, it is straightforward to obtain $j_{x \text {,ion }}$-whose dependence on $E_{x}$ is shown in Fig. 2(b) - by linear regression. For the range of $E_{x}$ studied, we see that the response is remarkably linear. Moreover, the data appear essentially free from noise, which is to be contrasted with the estimate of the current density from,

$$
\left\langle j_{x, \text { ion }}\right\rangle=\frac{1}{\Omega} \sum_{i}^{N_{\text {ion }}} q_{i}\left\langle v_{x, i}\right\rangle,
$$

where $v_{x, i}$ is the $x$-component of the $i^{\text {th }}$ ion's velocity, which is also shown in Fig. 2 (b). Although the general agreement with the estimate based on $\partial_{t} P_{x, \text { ion }}$ is sound, the degree of noise is far higher. This is not unexpected, as we are effectively attempting to extract the drift velocities imparted on the ions by the field. The $v_{x, i}$ that enter the average in Eq. 43 are instantaneous velocities, and are thus distributed according to the Maxwell-Boltzmann distribution. This gives rise to a relatively large error on each individual measurement. Conversely, we measure $P_{x, \text { ion }}$ at regular time intervals that are long (e.g. every $100 \mathrm{ps}$ ) compared to typical velocity autocorrelation times. This effectively averages out the Maxwell-
Boltzmann distribution, and greatly reduces the error in the estimate of the drift velocity, and therefore also the current density.

The clear linear response of $\left\langle j_{x, \text { ion }}\right\rangle$ seen in Fig. 2 makes it simple to calculate $\sigma_{\text {ion }}(0)$, whose dependence on $c$ is shown in Fig. 3(a). It is interesting to observe that $\sigma_{\text {ion }}(0)$ exhibits a maximum in the conductivity at $c \approx 4$ M. In Fig. 3 (b) we show the molar ionic conductivities $\Lambda_{\text {ion }}=\sigma_{\text {ion }} / c$. According to Kohlrausch's law, for low concentrations $\Lambda_{\text {ion }}$ behaves as,

$$
\Lambda_{\text {ion }}=\Lambda_{\text {ion }}^{(0)}-\mathcal{K} c^{1 / 2},
$$

where $\Lambda_{\text {ion }}^{(0)}$ is the limiting value of $\Lambda_{\text {ion }}$ i.e., the molar conductivity at infinite dilution. $\mathcal{K}$ is a system-dependent constant, which accounts for both electrophoretic and relaxation effects that impede the ionic motion. ${ }^{58} \mathrm{Al}-$ though we only have limited data at low $c$, fitting Eq. 44 for $c \lesssim 0.4 \mathrm{M}$ and extrapolating $c \rightarrow 0$ gives $\Lambda_{\text {ion }}^{(0)}=$ $97 \pm 2 \mathrm{~ns}^{-1} \mathrm{M}^{-1}$. This is to be contrasted with the experimental value of $\Lambda_{\text {ion }}^{(0)}=114 \mathrm{~ns}^{-1} \mathrm{M}^{-1} .59$ Given the simple point charge models used and the limited data at low $c$, this is a remarkably satisfactory level of agreement.

In addition to the ionic conductivities, our simulations also allow us to directly measure $\epsilon_{\text {wat }}$ via the constitutive relation Eq. 4. In Fig. 3 (c), $\left\langle\mathbf{P}_{\text {wat }}\right\rangle$ vs $\mathbf{E}$ is shown for all concentrations studied. As well as exhibiting a concentration dependence, the response is also noticeably non-linear. Nevertheless, the simulation data are well approximated by a third order polynomial, $\left\langle P_{x, \text { wat }}\right\rangle=$ $\left\langle P_{x, \text { wat }}\right\rangle_{0}+\chi_{\text {eff }}^{(1)} E_{x}+\chi_{\text {eff }}^{(2)} E_{x}^{2}+\chi_{\text {eff }}^{(3)} E_{x}^{3}$, and we can extract the solvent dielectric constant from the linear coefficient $\chi_{\mathrm{eff}}^{(1)}$,

$$
\chi_{\mathrm{eff}}^{(1)}=\frac{\epsilon_{\mathrm{wat}}-1}{4 \pi}
$$



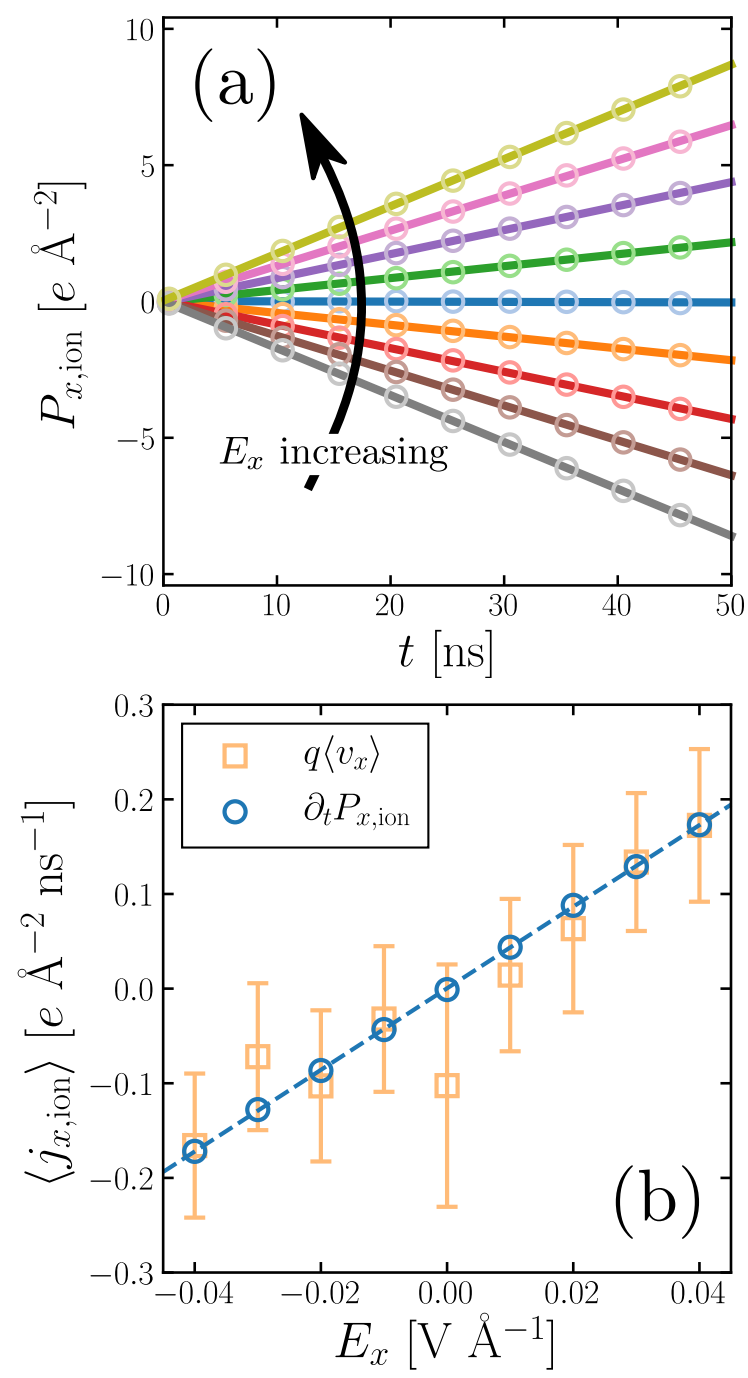

FIG. 2. Ionic response of a $1 \mathrm{M} \mathrm{NaCl}$ solution to a finite $\mathbf{E}$

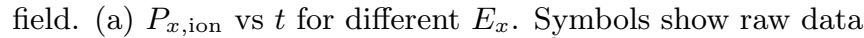
from the simulations (only every $100^{\text {th }}$ data point is shown for clarity), while solid lines show linear fits. The slope of each line gives the average ionic current density $\left\langle j_{x, \text { ion }}\right\rangle=$ $\left\langle J_{x, \text { ion }}\right\rangle / \Omega$ for that field strength. (b) $\left\langle j_{x \text {,ion }}\right\rangle$ vs $E_{x}$ obtained from the time evolution of $P_{x \text {,ion }}$ (blue circles). The error estimate is smaller than the size of the symbols. The dashed line shows a linear fit; it is evident that for the range of $E_{x}$ used, we are in a linear response regime. The orange squares show $\left\langle j_{x \text {,ion }}\right\rangle$ obtained from Eq. 43 , which exhibits a far higher degree of noise.

The concentration dependence of $\epsilon_{\text {wat }}$ obtained in this manner is shown in Fig. 3(d). Also shown is the static dielectric constant obtained from the fluctuations of the total solvent dipole moment,

$$
\epsilon_{\text {fluct }}-1=\frac{4 \pi \beta}{\Omega}\left\langle\left(\delta M_{x, \text { wat }}\right)^{2}\right\rangle
$$

Both $\epsilon_{\text {wat }}$ and $\epsilon_{\text {fluct }}$ depend on $c$ in a similar fashion, and
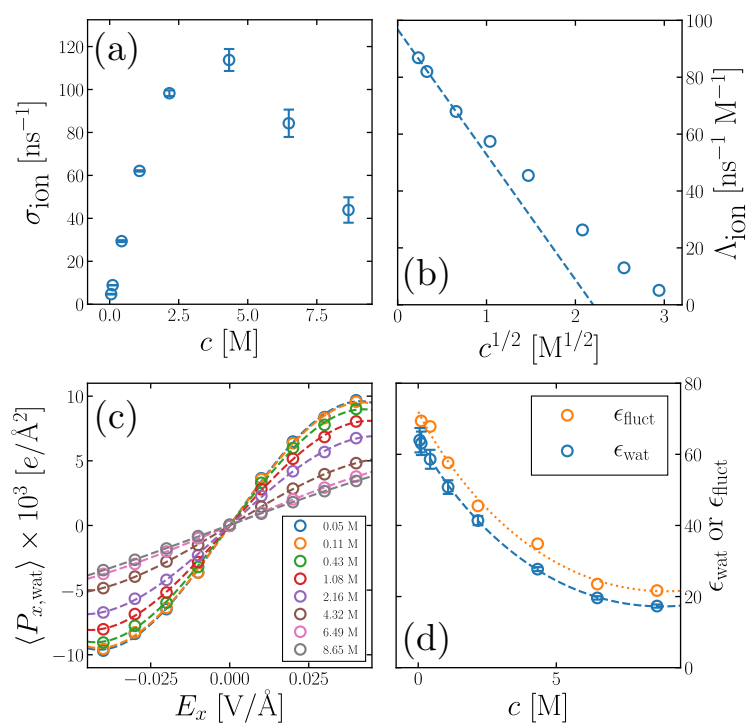

FIG. 3. (a) $\sigma_{\text {ion }}$ vs $c$. A maximum is observed at $c \approx 4 \mathrm{M}$. (b) $\Lambda_{\text {ion }}$ vs $c^{1 / 2}$. Kohlrausch's law is obeyed at low concentrations. (c) $\left\langle P_{x, \text { wat }}\right\rangle$ vs $E_{x}$ for different $c$ (see legend). The water responds non-linearly and also shows a $c$ dependence. The dashed lines show fits to third order polynomials. (d) $\epsilon_{\text {wat }}$ and $\epsilon_{\text {fluct }}$ vs $c$. Both measures of the dielectric constant are well described by the form $\epsilon=\epsilon_{0}-A c+B c^{3 / 2}$, although $\epsilon_{\text {wat }}$ (dashed line) is systematically lower than $\epsilon_{\text {fluct }}$ (dotted line). Data for $c=0.05 \mathrm{M}$ has been omitted due to inadequate statistics [panel (d) only].

are well approximated by the commonly used form, ${ }^{60,61}$

$$
\epsilon(c)=\epsilon_{0}-A c+B c^{3 / 2} .
$$

However, it is clear that $\epsilon_{\text {fluct }}$ is systematically higher than $\epsilon_{\text {wat }}$ across all concentrations. As discussed in the introduction, this difference is a direct measure of the dynamic contribution to the dielectric decrement. ${ }^{29-34}$ As shown by Caillol et al. ${ }^{16}$ the dynamic contribution is due to a coupling between the ionic current and solvent polarization,

$\lim _{\omega \rightarrow 0} \epsilon_{\text {wat }}(\omega)-\epsilon_{\text {fluct }}=\frac{4 \pi \beta}{3 \Omega} \int_{0}^{\infty} \mathrm{d} \tau\left\langle\delta \mathbf{M}_{\text {wat }}(\tau) \cdot \delta \mathbf{J}_{\text {ion }}(0)\right\rangle$.

Based on simulations between 1.8 and $2.2 \mathrm{~ns}$, and using comparable system sizes to those in this article, Chandra concluded that the dynamic contribution for aqueous $\mathrm{NaCl}$ is finite but small: Approximately two orders of magnitude smaller than the equilibrium contribution. ${ }^{34}$ To give a sense of the dynamic contribution obtained in this work, in the limit $c \rightarrow 0, \epsilon_{\text {fluct }}$ is found to be $72 \pm 3$, in excellent agreement with previously computed values of the the dielectric constant of SPC/E water. ${ }^{11,12,62-65}$ In contrast, the corresponding result for $\epsilon_{\mathrm{wat}}$ is $65 \pm 1$, i.e. roughly $10 \%$ smaller than $\epsilon_{\text {fluct }}$. Moreover, from Fig. $3(\mathrm{~d})$, it appears that the concentration dependence of the dynamic contribution is weak, and thus becomes proportionally more significant at higher concentrations. 
While our results are consistent with Chandra's observation that the dynamic contribution is finite, we conjecture that the differences in magnitude are due to difficulties in converging the long time contributions to the time correlation function in Eq. 48, see Fig. S9. Our finding of a larger dynamic contribution is also broadly consistent with Sega et $a l,{ }^{26,28}$ who used a nonequlibrium approach in which a fictitious field was applied only to the ions. However, these authors also reported a dependence on the force field used. We would like to stress that we have not attempted to evaluate any potential effects of finite system size, and we have extrapolated to infinite dilution from our relatively small simulation cells. What our results demonstrate is an alternative approach to investigating subtle effects such as the dynamic coupling between the solvent and ions, based on a Hamiltonian for a system at constant Maxwell field, E.

\section{B. Response to constant D}

In contrast to its constant $\mathbf{E}$ counterpart, the formulation of the constant $\mathbf{D}$ ensemble for finite temperature molecular dynamics simulations is a more recent development. This statement, however, warrants some qualification. In particular, if we set $\mathbf{D}=0$ in Eq. 19, then the forces derived from $\mathcal{H}_{\mathbf{D}}$ take the same form as those presented by CLW ${ }^{16-18}$ for an electrolyte solution surrounded by a medium with dielectric constant $\epsilon^{\prime}=0$. Moreover, if we only set the displacement field along one direction, say $D_{z}=0$, and use tin foil boundary conditions in the other two directions (so-called 'hybrid' boundary conditions ${ }^{11}$ ), then we recover the popular Yeh-Berkowitz (YB) correction for simulations in a slab geometry. ${ }^{66}$ Whereas in the YB scheme it is necessary to introduce a vacuum region, no such constraints are imposed on the system by $\mathcal{H}_{\mathbf{D}}$, a fact that was recently exploited in Refs. 13 in the study of electrolyte/solid interfaces. In this study, we have removed all extended interfaces entirely.

Despite the above similarities to the work of CLW and YB, $\mathcal{H}_{\mathbf{D}}$ has only recently been identified as the Hamiltonian for finite temperature MD simulation in the constant $\mathbf{D}$ ensemble. It is therefore unsurprising that the response of bulk electrolyte solutions to finite displacement fields has not been widely studied. In Fig. 4 we show $\left\langle P_{x \text {,ion }}\right\rangle$ and $\left\langle P_{x \text {,wat }}\right\rangle$ vs $D_{x}$ for different concentrations. Clearly, all the response originates from the ions, consistent with the discussion presented in Sec. II B. The dashed line in Fig. 4 (a) shows the theoretical result for a conductor, $4 \pi\left\langle P_{x \text {,ion }}\right\rangle=D_{x}$, to which the simulation data conforms excellently. We therefore conclude, as expected, that $\left\langle E_{x}\right\rangle=0$ in our simulation, which was the starting point for the derivation of the SL conditions (see Sec. II B). Do the simulations also confirm the quantitative theoretical predictions of the SL conditions given by Eqs. 34 and 41? This is indeed the case, as demonstrated in Fig. 5. From Fig. 5 (a), the expected anti- correlation of ionic and water polarization fluctuations is observed. In both Figs. 4 and 5 (a), we have omitted data for $c \lesssim 0.4 \mathrm{M}$, owing to insufficient statistics for the lowest concentrations (see below). Thus while the polarization fluctuations appear to decrease with $c^{1 / 2}$ in Fig. 5 (a), we cannot preclude deviations from this behavior at low concentration. Fig. 5 (b) shows the left hand sides of Eqs. 34 and 41, as measured from simulation. They are clearly consistent with the theoretical predictions; gathering statistics from all simulations gives,

$$
\frac{4 \pi \beta}{\Omega}\left[\left\langle\left(\delta M_{x, \text { ion }}\right)^{2}\right\rangle+\left\langle\left(\delta M_{x, \text { ion }}\right)\left(\delta M_{x, \text { wat }}\right)\right\rangle\right]=1.07 \pm 0.11
$$

$\frac{4 \pi \beta}{\Omega}\left[\left\langle\left(\delta M_{x, \text { wat }}\right)^{2}\right\rangle+\left\langle\left(\delta M_{x, \text { ion }}\right)\left(\delta M_{x, \text { wat }}\right)\right\rangle\right]=-0.05 \pm 0.11$.

Combined with the theoretical results of Sec. IIB, these simulation results are a powerful demonstration that, in a bulk electrolyte, fluctuations in the ionic and solvent polarization are inextricably linked.

As mentioned in the introduction, the finite field method for constant $\mathbf{D}$ was previously used by Pache and Schmidt to calculate $\epsilon_{\text {wat }}$ from the change in the polarization. ${ }^{36}$ This was done by coupling the $\mathbf{D}$ field only to the water polarization, which was motivated by the fact that, although a transient water polarization was observed when coupling the $\mathbf{D}$ to the total polarization, only the ions contributed upon reaching equilibrium. From the theoretical and simulation results presented here, this can be understood as a manifestation of the SL conditions. Applying the $\mathbf{D}$ field only to the water will affect the fluctuations and likely violate the SL conditions. Developing optimal strategies for computing $\epsilon_{\text {wat }}$ for electrolyte systems from constant $\mathbf{D}$ simulations requires further theoretical considerations that lie beyond the scope of the current article.

We end this section with a comment regarding the timescales for relaxation toward equilibrium. In Fig. 6 we show the time evolution of the total polarization $P_{x}$, along with its contributions $P_{x \text {,wat }}$ and $P_{x \text {,ion }}$, for $c \approx 0.11 \mathrm{M}$ and $D_{x}=2.0 \mathrm{~V} / \AA$. It is clear that while $P_{x}$ attains its equilibrium value relatively quickly, $P_{x, \text { wat }}$ and $P_{x \text {,ion }}$ take on the order of 1 ns to relax. It is also apparent that there exists correlations over long timescales

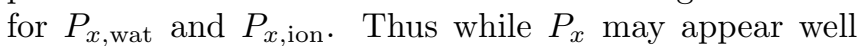
converged on short timescales, there is a real risk of inadequate sampling of the equilibrium phase space distribution function. Although such effects are exaggerated for low concentrations, these results are potentially concerning for $a b$ initio MD studies of electrolyte systems at constant $\mathbf{D}$, but may guide future strategies for tackling such issues. 

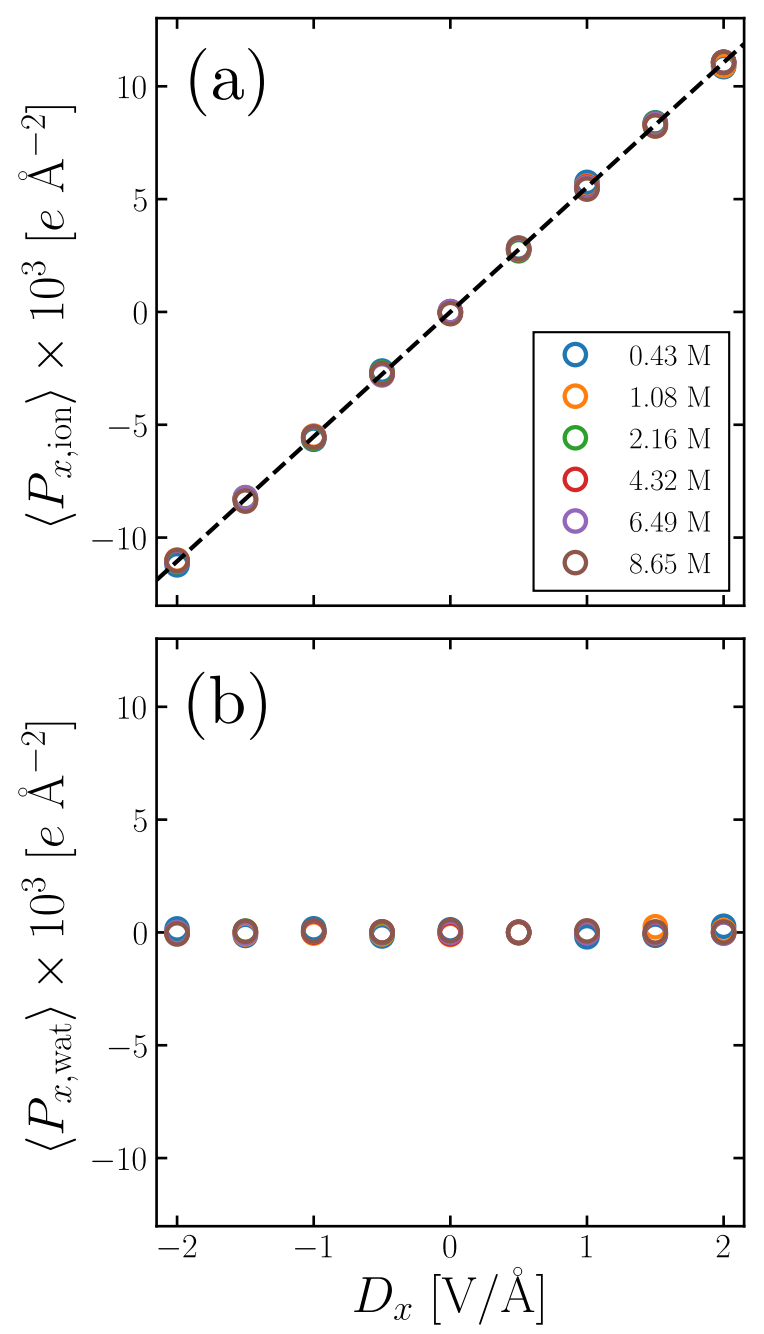

FIG. 4. The ions completely screen the $\mathbf{D}$ field. (a) $P_{x \text {,ion vs }}$ $D_{x}$ for different $c$, as indicated by the legend. The polarization response from the ions is independent of $c$. The dashed line shows $P_{x \text {,ion }}=D_{x} / 4 \pi$, the theoretical result for a conductor. (b) $P_{x \text {,wat }}$ vs $D_{x}$. There is negligible solvent response. Error estimates are smaller than the size of the symbols. Data for $c \lesssim 0.4 \mathrm{M}$ has been omitted due to inadequate statistics.

\section{SUMMARY}

The purpose of this work was to investigate the behavior of bulk aqueous electrolyte solutions using the finite field methods developed in Refs. 11-13. In comparison to existing theoretical frameworks, this has offered great conceptual simplifications. Using the Hamiltonian for constant Maxwell field E, we derived the linear response formula for the ionic conductivity without reference to the vector potential. The particularly pleasing aspect of this approach is that the Hamiltonian used to derive the linear response relation is the same as that used to derive the forces for molecular dynamics simulations. This was put into practice here to obtain an ionic conductivity at infinite dilution within $15 \%$ of experimental values.
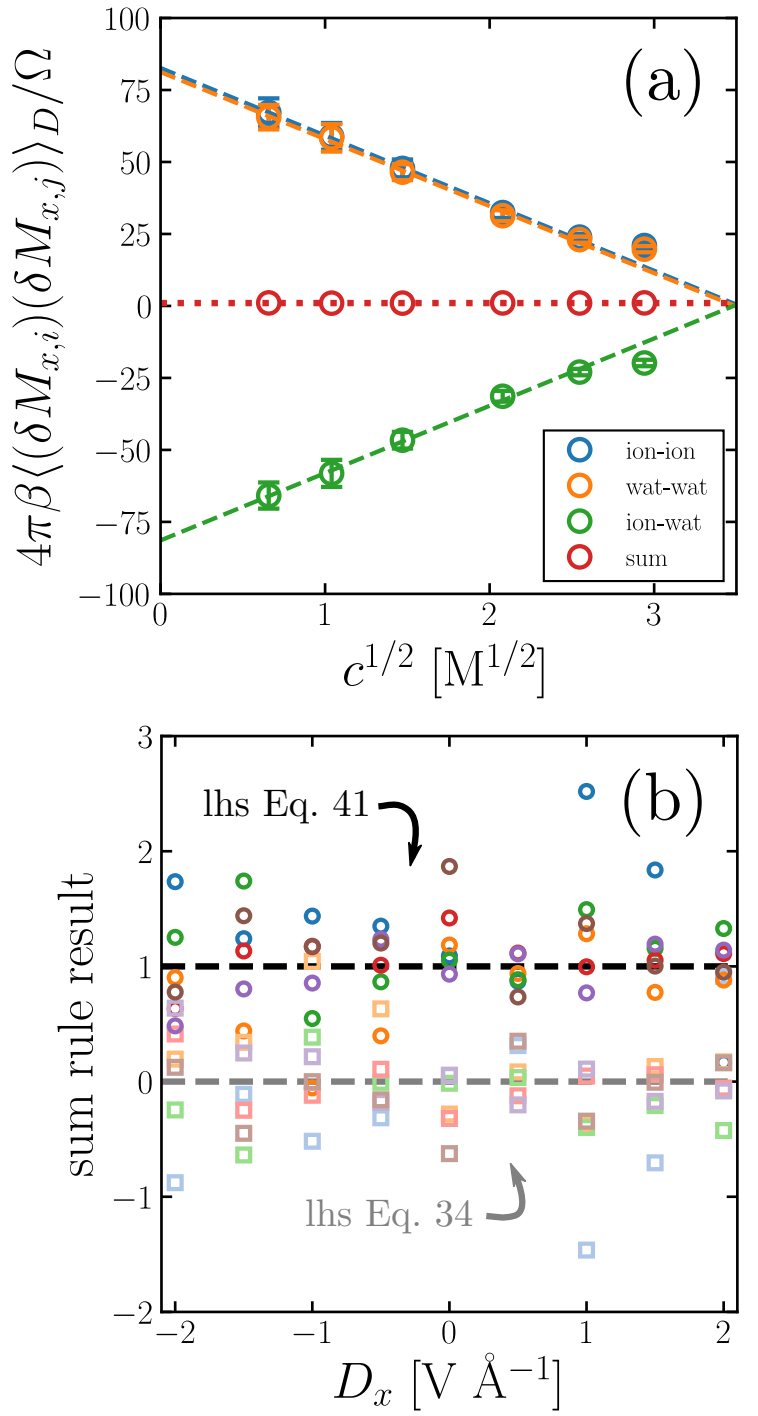

FIG. 5. The Stillinger-Lovett conditions place strict requirements on the polarization fluctuations.

(a) $4 \pi \beta\left\langle\left(\delta M_{x, i}\right)\left(\delta M_{x, j}\right)\right\rangle$ vs $c^{1 / 2}$, with $i, j=$ 'ion' or 'wat'. Both $\left\langle\left(\delta M_{x, \text { ion }}\right)^{2}\right\rangle$ (blue) and $\left\langle\left(\delta M_{x} \text {,wat }\right)^{2}\right\rangle$ (orange) decrease approximately linearly with $c^{1 / 2}$. Conversely, $\left\langle\left(\delta M_{x, \text { ion }}\right)\left(\delta M_{x, \text { wat }}\right)\right\rangle$ increases (green), such that the sum rule, Eq. 22, is satisfied (red). (b) The left hand sides of Eq. 34 (squares) and Eq. 41 (circles); the black and gray dashed lines show the respective theoretical predictions. Different colors refer to different $c$, as in Fig. 4. Data for $c \lesssim 0.4 \mathrm{M}$ has been omitted due to inadequate statistics.

In addition, this approach enabled us to extract the dielectric constant of the solvent water from its response to finite $\mathbf{E}$, which was seen to decrease with increasing electrolyte concentration. We also observed that the dielectric constant measured from the response was systematically smaller than that estimated from fluctuations of the solvent polarization, which can be taken as a direct measure of the dynamic coupling between fluctuations of the solvent polarization and ionic current. 


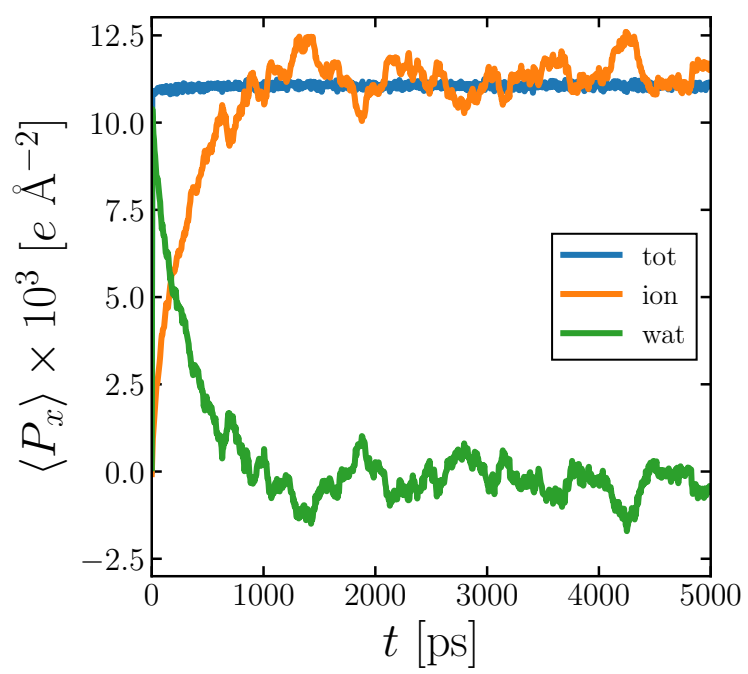

FIG. 6. Time evolution of the total polarization $P_{x}$, and its contributions from the water and ions $\left(P_{x, \text { wat }}\right.$ and $P_{x \text {,ion }}$, respectively), for $c \approx 0.11 \mathrm{M}$ and $D_{x}=2.0 \mathrm{~V} / \AA$. The initial configuration was taken from an equilibrated $\mathbf{D}=\mathbf{0}$ simulation. While $P_{x}$ attains its equilibrium value relatively quickly, $P_{x \text {,wat }}$ and $P_{x \text {,ion }}$ take much longer to relax (approx. $1 \mathrm{~ns}$ ), and also exhibit correlations over long timescales.

We also used the finite field method for constant electric displacement $\mathbf{D}$ to derive the Stillinger-Lovett conditions that relate ionic and solvent polarization fluctuations. In addition to providing a mechanical picture with which to understand the SL conditions, this approach avoids the need to relate the perturbing field to the cavity field. We exploited this fact in our simulations to explicitly measure the system's response to finite $\mathbf{D}$, which supported the theoretical predictions. At equilibrium, we found that all polarization response emanates from the ions. We also observed that relaxation of the ionic polarization to equilibrium could be a slow process, especially for dilute solutions. The anticorrelations imposed by the Stillinger-Lovett conditions, however, causes the solvent polarization to relax in a manner such that the total polarization appears to attain its equilibrium value on relatively short timescales.

One of the major motivations for the development of the finite field methods for finite temperature simulations was to mitigate spatial finite size effects that lead to incomplete screening of charged insulator/electrolyte interfaces. While we remain optimistic that such techniques will prove a useful tool in ab initio studies of such systems, our results emphasize the need to exercise caution with respect to proper sampling of the equilibrium phase space distribution function. Our results also showcase the application of different electrostatic boundary conditions to electrolyte systems beyond the slab geometry employed for solid-liquid interfaces. The finite field methods can therefore be viewed as an additional tool with which to study electrolyte systems, and may find uses in e.g. the computation of dielectric spectra, ${ }^{23-28}$ or ion transport through membranes. ${ }^{55-57}$ The key challenge faced now is to generalize this approach to systems with applied electric fields and polarization varying in space. In other words, how to define polarization density for the ions for which recourse to the multipole expansion is not possible?

\section{SUPPLEMENTARY MATERIAL}

See supplementary material for results obtained with concentration dependent simulation cell sizes such that the pressure remained constant, results obtained with hybrid boundary conditions, and a comparison to the Green-Kubo approach.

\section{ACKNOWLEDGMENTS}

Peter Wirnsberger is thanked for many helpful discussions, especially regarding implementation of the constant-D ensemble in LAMMPS. Thomas Sayer is thanked for his comments on a draft of this manuscript. We are grateful for computational support from the UK Materials and Molecular Modelling Hub, which is partially funded by EPSRC (EP/P020194), for which access was obtained via the UKCP consortium and funded by EPSRC grant ref EP/P022561/1. S.J.C. is supported by a Royal Commission for the Exhibition of 1851 Research Fellowship.

${ }^{1}$ Y. Zhang and P. S. Cremer, Curr. Opin. Chem. Bio. 10, 658 (2006).

${ }^{2}$ Y. Min, M. Akbulut, K. Kristiansen, Y. Golan, and J. Israelachvili, Nature Mater. 7, 527 (2008).

${ }^{3}$ G. B. Sukhorukov, A. A. Antipov, A. Voigt, E. Donath, and H. Möhwald, Macromol. Rapid Commun. 22, 44 (2001).

${ }^{4}$ J. Kolny, A. Kornowski, and H. Weller, Nano Lett. 2, 361 (2002).

${ }^{5}$ J. Simard, C. Briggs, A. K. Boal, and V. M. Rotello, ChemComm , 1943 (2000).

${ }^{6}$ N. Duff, Y. R. Dahal, J. D. Schmit, and B. Peters, J. Chem. Phys. 140, 014501 (2014).

${ }^{7}$ T. F. Whale, M. A. Holden, T. W. Wilson, D. O'Sullivan, and B. J. Murray, Chem. Sci. 9, 4142 (2018).

${ }^{8}$ G. J. Morris and E. Acton, Cryobiology 66, 85 (2013).

${ }^{9}$ L. Xia, L. Yu, D. Hu, and G. Z. Chen, Mater. Chem. Front. 1, 584 (2017).

${ }^{10}$ M. Stengel, N. A. Spaldin, and D. Vanderbilt, Nature Phys. 5, 304 (2009).

${ }^{11}$ C. Zhang and M. Sprik, Phys. Rev. B 93, 144201 (2016).

${ }^{12}$ C. Zhang, J. Hutter, and M. Sprik, J. Phys. Chem. Lett. 7, 2696 (2016).

${ }^{13}$ C. Zhang and M. Sprik, Phys. Rev. B 94, 245309 (2016).

${ }^{14}$ T. Sayer, C. Zhang, and M. Sprik, J. Chem. Phys. 147, 104702 (2017).

${ }^{15}$ T. Sayer, M. Sprik, and C. Zhang, J. Chem. Phys. 150, 041716 (2019).

${ }^{16}$ J.-M. Caillol, D. Levesque, and J. J. Weis, J. Chem. Phys. 85, 6645 (1986)

${ }^{17}$ J.-M. Caillol, D. Levesque, and J. J. Weis, J. Chem. Phys. 91, 5544 (1989).

${ }^{18}$ J.-M. Caillol, D. Levesque, and J. J. Weis, J. Chem. Phys. 91, 5555 (1989).

${ }^{19}$ F. H. Stillinger Jr and R. Lovett, J. Chem. Phys. 48, 3858 (1968). 
${ }^{20}$ F. H. Stillinger Jr and R. Lovett, J. Chem. Phys. 49, 1991 (1968).

${ }^{21} \mathrm{P}$. Madden and D. Kivelson, "A consistent molecular treatment of dielectric phenomena," in Adv. Chem. Phys. (John Wiley \& Sons, Inc., 1984) pp. 467-566.

${ }^{22}$ R. L. Fulton, J. Chem. Phys. 68, 3095 (1978).

${ }^{23}$ C. Schröder, M. Haberler, and O. Steinhauser, J. Chem. Phys. 128, 134501 (2008).

${ }^{24}$ C. Schröder, J. Hunger, A. Stoppa, R. Buchner, and O. Steinhauser, J. Chem. Phys. 129, 184501 (2008).

${ }^{25}$ M. Sega, S. S. Kantorovich, A. Arnold, and C. Holm, in Recent Advances in Broadband Dielectric Spectroscopy (Springer, 2013) pp. $103-122$.

${ }^{26}$ M. Sega, S. S. Kantorovich, C. Holm, and A. Arnold, J. Chem. Phys. 140, 211101 (2014)

${ }^{27}$ K. F. Rinne, S. Gekle, and R. R. Netz, J. Chem. Phys. 141, 214502 (2014).

${ }^{28}$ M. Sega, S. Kantorovich, and A. Arnold, Phys. Chem. Chem. Phys. 17, 130 (2015).

${ }^{29}$ J. Hubbard, L. Onsager, W. Van Beek, and M. Mandel, Proc. Natl. Acad. Sci. USA 74, 401 (1977).

${ }^{30}$ J. Hubbard and L. Onsager, J. Chem. Phys. 67, 4850 (1977).

${ }^{31}$ J. B. Hubbard, P. Colonomos, and P. G. Wolynes, J. Chem. Phys. 71, 2652 (1979).

${ }^{32}$ B. Felderhof, Mol. Phys. 51, 801 (1984).

${ }^{33}$ A. Chandra, D. Wei, and G. Patey, J. Chem. Phys. 98, 4959 (1993).

${ }^{34}$ A. Chandra, J. Chem. Phys. 113, 903 (2000).

${ }^{35}$ J.-M. Caillol, J. Chem. Phys 101, 6080 (1994).

${ }^{36}$ D. Pache and R. Schmid, ChemElectroChem 5, 1444 (2018).

${ }^{37}$ With the Fourier transform defined by Eq. 6 , the complex susceptibility can be expressed as $\chi(\omega)=\chi^{\prime}(\omega)+i \chi^{\prime \prime}(\omega)$, where $\chi^{\prime}$ and $\chi^{\prime \prime}$ denote the real and imaginary parts of $\chi$, respectively. This follows, e.g. Refs. 21 and 41. Another convention often seen in the literature is to define the Fourier transform with a minus sign in the exponent. In this case, $\chi(\omega)=\chi^{\prime}(\omega)-i \chi^{\prime \prime}(\omega)$.

${ }^{38}$ M. Sprik, Mol. Phys. 116, 3114 (2018).

${ }^{39}$ E. A. Power and S. Zienau, Phil. Trans. Royal Soc. A 251, 427 (1959).

${ }^{40}$ L. D. Landau, E. M. Lifshitz, and L. P. Pitaevskii, Electrodynamics of Continuous Media, 2nd ed. (Butterworth-Heinemann, 1984).
${ }^{41}$ J.-P. Hansen and I. R. McDonald, Theory of Simple Liquids, 4th ed. (Academic Press, 2013).

${ }^{42}$ L. Blum, C. Gruber, J. L. Lebowitz, and P. Martin, Phys. Rev. Lett. 48, 1769 (1982).

${ }^{43}$ P. A. Martin, Rev. Mod. Phys. 60, 1075 (1988).

${ }^{44}$ S. L. Carnie, J. Chem. Phys. 78, 2742 (1983).

${ }^{45}$ J. M. Caillol, J. Chem. Phys. 96, 7039 (1992).

${ }^{46}$ H. J. C. Berendsen, J. R. Grigera, and T. P. Straatsma, J. Phys. Chem. 91, 6269 (1987)

${ }^{47}$ I. S. Joung and T. E. Cheatham III, J. Phys. Chem. B 112, 9020 (2008).

${ }^{48}$ S. Plimpton, J. Comput. Phys. 117, 1 (1995).

${ }^{49}$ R. W. Hockney and J. W. Eastwood, Computer simulation using particles (CRC Press, 1988).

${ }^{50}$ J. Kolafa and J. W. Perram, Mol. Sim. 9, 351 (1992).

${ }^{51}$ W. Shinoda, M. Shiga, and M. Mikami, Phys. Rev. B 69, 134103 (2004).

52 M. E. Tuckerman, J. Alejandre, R. López-Rendón, A. L. Jochim, and G. J. Martyna, J. Phys. A 39, 5629 (2006).

${ }^{53}$ H. C. Andersen, J. Comput. Phys. 52, 24 (1983).

${ }^{54}$ The source code is freely available at https://github.com/ uccasco/FiniteFields.

${ }^{55}$ B. Roux, T. Allen, S. Berneche, and W. Im, Q. Rev. Biophys. 37, 15 (2004).

${ }^{56}$ N. Modi, M. Winterhalter, and U. Kleinekathoefer, Nanoscale 4, 6166 (2012)

${ }^{57}$ C. Maffeo, S. Bhattacharya, J. Yoo, D. Wells, and A. Aksimentiev, Chem. Rev. 112, 6250 (2012).

${ }^{58}$ J. O. Bokris and A. K. N. Reddy, Modern Electrochemistry 1, 2nd ed. (Plenum Press, 1998).

${ }^{59}$ G. W. C. Kaye and T. H. Laby, Tables of physical and chemical constants (Longman, London, 1973).

${ }^{60}$ H. L. Friedman, J. Chem. Phys. 76, 1092 (1982).

${ }^{61}$ W. R. Fawcett, Liquids, Solutions, and Interfaces (Oxford University Press, 2004).

${ }^{62}$ D. van der Spoel, P. J. van Maaren, and H. J. Berendsen, J. Chem. Phys. 108, 10220 (1998)

${ }^{63}$ J. Aragones, L. MacDowell, and C. Vega, J. Phys. Chem. A 115, $5745(2010)$

${ }^{64}$ C. Zhang and G. Galli, J. Chem. Phys. 141, 084504 (2014).

${ }^{65}$ D. Braun, S. Boresch, and O. Steinhauser, J. Chem. Phys. 140, 064107 (2014).

${ }^{66}$ I.-C. Yeh and M. L. Berkowitz, J. Chem. Phys. 111, 3155 (1999). 